\title{
El cuidado del paciente terminal
}

\section{Care of the terminal patient}

\author{
R. Buisán ${ }^{1}$ J. C. Delgado²
}

\section{RESUMEN}

En el ejercicio de las profesiones sanitarias siempre ha habido quienes creían que lo importante era tratar a todos los pacientes hasta sus últimas consecuencias, pero también quienes han defendido que lo importante era tratar y cuando esto no era posible, cuidar a las personas hasta el final. Entre éstas se encontraba Cicely Saunders, fundadora del movimiento de los hospices. Ella defendía que en la fase terminal de la enfermedad, cuando el deterioro que va a sufrir el enfermo es cada vez mayor y por lo tanto, muy impactante también para la familia y el equipo que le trata, el objetivo debe cambiar y ser sustituido por el cuidado como única meta. Ese cuidado debe virar hacia proporcionar confort al enfermo y a la familia. Porque en esta fase, cada acción positiva realizada sobre uno de ellos es tomada como algo también positivo por el otro.

El equipo sanitario debe transformarse en un equipo cuidador. El cuidado como medio y la calidad de vida y el confort como finalidad son los objetivos que debemos tener los cuidadores durante la fase final de la vida, tal y como promulgó el Subcomité Europeo de Cuidados Paliativos de la CEE, el 5 de mayo de 1991, al definir los cuidados paliativos.

Palabras Clave. Cuidado. Enfermería. Cuidados Paliativos. Bioética.

An. Sist. Sanit. Navar. 2007; 30 (Supl. 3): 103-112.

\section{ABSTRACT}

In the work of health professions there have always been those who believed that the important thing was to treat all the patients until the final consequences, but there are also those who have considered that the important thing was to treat the patient and when this was not possible, to care for him until the end. Amongst the latter was Cicely Saunders, founder of the Hospices movement. She believed that in the terminal phase of the disease, when there was an increase in the deterioration the patient was to suffer, which also had a great impact on the family and team treating him/her, the aim should change and be replaced by care as the only goal. That care should be redirected towards providing comfort to the patient and his/her family. Because in this phase each positive action carried out on one of them is taken as something that is also positive for the other.

The health team must transform itself into a care team. Care as a means and the quality of life and comfort as an end are must be our aims as carers during the final phase of life, as announced by the European Sub-committee of Palliative Care of the EEC, May $5^{\text {th }} 1991$, when defining palliative care.

Key words. Care. Infirmary. Palliative Care. Bioethics.
1. Enfermera. Experta en Bioética.

2. Enfermero. Antropólogo. Experto en Bioética.

\section{Correspondencia:}

Dña. Raquel Buisán

AE Federica Montseny

Avef. La Albufera, 285

28038 Madrid

e-mail: jcantolin@terra.es 


\section{INTRODUCCIÓN}

Desde los años cincuenta del pasado siglo, la medicina y por analogía sus otras hermanas sanitarias como puede ser la enfermería tomaron la vía del desarrollo técnico. En un principio parecía el camino correcto y seguramente lo sea, pues este camino de desarrollo técnico y de enormes avances científicos ha proporcionado que enfermedades incurables o no tratables hasta ese momento, fueran controlables, tratables, cronificables e incluso curables. No cabe duda que las unidades de cuidados intensivos (UCI) han conseguido salvar muchas vidas y que los avances contra el cáncer o las enfermedades infecciosas, han permitido vivir a personas que tenían anteriormente dictada una sentencia de muerte o un desahucio medico incuestionable. Esos avances han permitido que las personas vivan más y a veces mejor, pues la evolución natural de ciertos procesos patológicos era insidiosa, prolongada y muchas veces agónica. Pero también algunas veces a proporcionado que se hicieran cosas que no deberían de hacerse, prolongando la vida o quizás la agonía a ciertas personas en el final de su proceso patológico. A los que llevamos ya algunos años en este mundo sanitario no nos resultan extrañas frases como: "Hay que hacer todo lo posible por...", "Vamos a subirle a la UCI, que mientras hay vida hay esperanza", "Haga todo lo posible para que no se muera...", "No responde a la quimioterapia, pero como no podemos hacer otra cosa vamos a ponerle otro ciclo". También es verdad que había otras frases que también sonaban a la vez: "Vamos a dejarle morir en paz", "Para que vamos a seguir con... Si ya no responde". Es decir, durante unos años había algunas personas que veían que lo importante era tratar a todos hasta sus últimas consecuencias y existían otros, que creían que lo importante era tratar y cuando esto no era posible, cuidar a las personas hasta el final.

Una de estas últimas personas sin duda fue Cicely Saunders, una enfermera inglesa que posteriormente estudio medicina, y fue la principal promotora del movimiento británico de hospice. Este tipo de establecimientos eran lugares donde se cuidaba a las personas próximas a la muerte; siendo este movimiento el precursor de los Cuidados Paliativos. Esta enfermera impresionada por como morían los soldados en la II Guerra Mundial, llenos de dolor y sufrimiento, se propuso realizar todo lo humanamente posible para acabar con la situación de sufrimiento de los enfermos moribundos y así, en 1967 inaugura el St. Christopher Hospice de Londres, después de haber trabajado en otros hospices de Inglaterra. Este hospital se pudo construir gracias a haber heredado una fuerte cantidad de dinero de David Tasma, un enfermo atendido por ella en fase terminal y que en agradecimiento al trato recibido en sus últimos momentos y viendo la labor que estaba realizando Cicely Saunders, la hizo partícipe de su fortuna personal.

Fue Cicely Saunders quien en los años setenta del siglo pasado propuso su teoría de "Total care" (Cuidado Total) y que posteriormente fue transformándose en "Total Confort" (Confort Total). Sin duda, ambas son dos maneras de concebir el cuidado de la persona de una forma global y no meramente como un control del dolor $u$ otros síntomas del enfermo en la fase final.

En un momento de agotamiento terapéutico como en el que nos encontramos en la enfermedad en fase terminal, donde el deterioro que va a sufrir el enfermo es cada vez mayor y por lo tanto, muy impactante para la familia y el equipo que le trata, nuestro objetivo debe cambiar y debe ser sustituido por el cuidado como único objetivo. Ese cuidado debe virar hacia proporcionar confort al enfermo y a la familia; no debemos olvidar que en nuestra cultura, el binomio paciente-familia siempre están muy interrelacionados en el mundo de la salud y la enfermedad, pero en el final de la vida se encuentran enormemente relacionados. Tal es así, que cada acción positiva realizada sobre uno de ellos es tomada como algo también positivo por el otro. Cuando tratamos de aliviar el dolor, la disnea, el apetito... del enfermo y obtenemos resultados satisfactorios, el familiar que cuida hasta la extenuación a dicho paciente también nota el alivio, pero no solamente en el plano psicológico, si no en el plano físico, pues el cuidar de su enfermo se hace más fácil y llevadero. Del mismo modo, cuando un 
paciente que es consciente de la sobrecarga que su enfermedad plantea a la familia, nota a alguien interesándose por el sufrimiento y las necesidades de su entorno familiar, lo percibe como una acción positiva para su cuidado, porque su familia ha encontrado un apoyo para sus necesidades.

El equipo sanitario debe transformarse en un equipo cuidador. Bien es verdad que para los profesionales de Enfermería el cuidado es su razón de ser, pero el resto de los profesionales deben convertirse en mayor medida en cuidadores, no en sanadores. El cuidado como medio y la calidad de vida y el confort como finalidad son los objetivos que debemos tener los cuidadores durante la fase final de la vida. Como muy bien definió el subcomité Europeo de Cuidados Paliativos de la CEE, el 5 de mayo de 1991, los Cuidados Paliativos son: "La asistencia total, activa y continuada de los pacientes y sus familias por un equipo multiprofesional cuando la expectativa médica no es la curación. La meta fundamental es dar calidad de vida al paciente y su familia sin intentar alargar la supervivencia. Debe cubrir las necesidades físicas, psicológicas, espirituales y sociales del paciente y su familia"1.

Dos son los aspectos del cuidado en los que nos vamos a detener como esenciales para dar una mayor calidad de vida a estas personas en su etapa final: La comunicación y las acciones directas sobre las necesidades alteradas. Vamos a hablar de ambas.

\section{LA COMUNICACIÓN COMO FORMA DE CUIDADO}

Cuando pensamos en los cuidados a una persona, siempre o casi siempre imaginamos acciones positivas, donde nuestros actos se materialicen de una forma real en el bienestar del paciente. Nuestra idea del cuidado suele ser procurar su alimentación, hidratación, descanso, etc, pero normalmente se nos olvida la importancia que tiene la comunicación en dicho cuidado, pudiendo ser la misma comunicación una forma de cubrir las necesidades de la persona enferma o afectada ${ }^{2}$.
Dentro de nuestro trabajo, se nos olvida con cierta frecuencia que a veces las herramientas más importantes son la palabra y la escucha. El establecer una comunicación abierta con el enfermo en fase terminal es para los cuidadores un escollo difícil de salvar en la práctica diaria. La muerte y el proceso de morir evocan en nosotros reacciones psicológicas que producen directa o indirectamente a evitar la comunicación. Nunca es fácil contestar a preguntas como: ¿Cuánto me queda de vida? ¿Cómo voy a morir? ¿Por qué a mí? Ante este tipo de preguntas nos quedamos sin respuesta y es que a veces no tienen respuesta. Como dice Bertrand Russell en Misticismo y Lógica: "Desde el punto de vista de la filosofía, el descubrimiento de que una pregunta carece de respuesta es una respuesta tan completa como cualquier otra".

Para Buber ${ }^{3}$ existen tres tipos de diálogos: El auténtico, el técnico y el monólogo ${ }^{4}$ Vamos a tratar de aclararlos.

- El diálogo auténtico. Es bidireccional, se aprende y se enseña, existe retroalimentación de la información y por lo tanto hay comunicación, es positivo para los interlocutores.

- El diálogo técnico. Es una escucha objetiva y es lo que se conoce como información unidireccional, uno habla y otro escucha. Suele asociarse a la información de expertos en algún tema. Los cuidadores caemos con frecuencia en él, pues nos falta la retroalimentación y saber que ha comprendido el paciente de lo que hemos dicho.

- El monólogo ${ }^{4}$, nosotros añadiríamos que compartido. Dos o más personas hablan siempre consigo mismo aunque piensen intercambiar información. Sólo sirve para desahogarse de las tensiones, como válvula de escape pero no existe intercambio de experiencias y conocimientos. No existe la escucha activa por ninguna de las partes.

La comunicación es todo un proceso de hacer partícipe a otro de lo que pienso y saber mediante el diálogo de lo que él piensa, por lo tanto es un proceso tanto activo, decir, como pasivo, escuchar, si es que podemos decir que escuchar es un 
proceso pasivo y en ambos, tenemos que poner toda nuestra atención. Por tanto, podríamos definir la comunicación como: "Hacer partícipe o transmitir a otra persona algo que se tiene, información, sentimientos, pensamientos". Lo que no se tiene no se puede transmitir.

En el cuidado de una persona ante la muerte, la comunicación es algunas veces, la mayor tecnología que podemos emplear. Por ella podemos realizar dos funciones de enorme importancia. Primera, informar, asesorar y enseñar, tanto al paciente como a la familia, modos y formar de un determinado tipo de cuidado. Segunda, informarnos, aprender y comprender cuales son las prioridades del paciente y de la familia. Cuales son sus temores, sus preferencias, sus valores y sobre todo que es lo bueno para ellos. El cuidado debe ser un concepto regido por la beneficencia y la beneficencia sólo se puede ejercer plenamente, cuando se sabe las preferencias del que se cuida, no del que cuida. Yo no puedo hacer el bien a alguien si no sé lo que es el bien para esa persona. Si esta idea es importante, cuando atendemos a una persona sana o enferma, es fundamental si esta se encuentra en la fase final de su vida. Jamás podremos cuidar bien y promocionar la beneficencia a una persona en fase terminal, si le impedimos que pueda ejercer su autonomía. Sus opiniones, deseos y preferencias servirán de guía para asesorarle, enseñarle y aconsejarle en los cuidados que se deben llevar a cabo.

Los cuidadores debemos integrar este concepto de la comunicación en nuestra relación, y no sólo con el enfermo, sino con la familia del enfermo. Ésta relación profesional que nos ocupa es una relación de ayuda y debe ser interpretada de forma especial.

La relación de ayuda plantea la existencia de dos partes distintas. Una parte débil compuesta por el enfermo y su familia, con múltiples malestares que pueden ser subjetivos u objetivos, pero que para ellos son reales, y una parte fuerte compuesta por el equipo cuidador, capaz de ayudar a solucionar parte de esos malestares. Es una relación asimétrica donde una de las par- tes necesita de la otra, donde una de las partes se encuentra en una situación de potencial debilidad respecto de la otra. Por eso, debemos tener cuidado a la hora de tomar decisiones para no afectar la autonomía del enfermo, pues ayudar y apoyar no implica hacer del otro sujeto un completo inválido, que no pueda ejercer su propia autonomía. En la comunicación se debe ser especialmente cuidadoso para no lesionar o cambiar las preferencias y los valores del paciente.

\section{ALGUNAS RECOMENDACIONES EN LA COMUNICACIÓN}

Los cuidadores vamos a tener que informarnos e informar muchas veces durante nuestra actividad cotidiana, esto a veces va a facilitar el hecho de comunicarnos, pues mientras nos encontramos distraídos e imbuidos en una actividad distinta del hecho de la conversación, puede facilitarnos mejores momentos para indagar en los temores, desilusiones y razones del enfermo y su familia. Mientras realizamos nuestras tareas y nuestras técnicas con el enfermo, puede ser un momento magnífico para hablar con él y con su familia, pero puede ser también un momento en que lo mismo que el enfermo baja la guardia para expresarnos sus problemas, nosotros también podemos decir cosas inadecuadas y dar información no recomendable. No debemos estar desprevenidos y estar atentos para dar siempre una información adecuada tanto para el enfermo como para la familia. Valgan las siguientes recomendaciones que pueden ayudarnos.

La comunicación no es un acto único. Debe ser gradual y paulatina. Es un proceso que va a durar durante toda la relación. No tenemos que asediarle a preguntas nada más iniciar nuestra actividad profesional con él. También se debe dosificar la información, no darla de golpe y en la primera entrevista, la situación seguro que va a ser cambiante y nuestra forma de enfrentarnos a los cambios van a modificar la información que demos.

Esperar a que pregunte $y$ hable el paciente. Conviene saber lo que sabe el paciente para no caer en errores o infor- 
maciones inadecuadas. Practicar la escucha activa. El paciente marca el camino y el ritmo; debemos seguir el criterio de la verdad soportable. El paciente irá preguntando lo que quiere saber y lo que puede soportar saber. Él nos irá dando la pauta a seguir, a veces, ante preguntas como: ¿Cuanto tiempo me queda de vida? Lo mejor es devolverla ¿por qué lo pregunta? La contestación nos dará la respuesta de lo que quiere realmente saber el paciente.

Saber usar los silencios. No hay que rellenar la entrevista de palabras. Hay que saber lo que hay que decir $y$, a veces, cuando no hay seguridad de saber lo que nos pregunta el enfermo, es mejor un "no sé”, que una larga explicación que no dice nada ni aclara las dudas del enfermo. Pero si el paciente no pregunta y el silencio se mantiene vez tras vez, debemos analizar si esto es por mutismo, por falta de empatía por nuestra parte, falta de confianza, etc.

Simplicidad y sin palabras "malsonantes". Debemos usar un lenguaje sencillo, sin tecnicismos, aclarando bien los conceptos y a la vez debemos evitar en nuestra conversación palabras o frases malsonantes como "cáncer", "muerte", "no hay tratamiento", "no puedo hacer nada más". El paciente puede emplear estas palabras y nos dará pistas de cual es el nivel de aceptación de su situación, pero si nosotros emitimos estos mensajes sin su consentimiento, se puede producir un bloqueo informativo y el paciente o la familia no escuchara nada más de lo que le digamos. Después de ciertas palabras se olvida hasta el $40 \%$ de lo que se dice después. Esto no implica que debamos mentir, no debemos mentir. La mentira siempre se puede volver en contra nuestra y si persistimos en ella, puede llegar un momento que sea difícil de mantener y al conocer la verdad perdamos la confianza del enfermo.

Por último, debemos usar correctamente el lenguaje no verbal. Durante la comunicación empleamos, a veces sin saberlo, todas nuestras artes para expresarnos y estas, no son sólo verbales. No debe basarse exclusivamente en lo que decimos, también importa como lo deci- mos, y los gestos y movimientos que empleamos al decirlo. Nuestra comunicación es un conjunto de contenido verbal, paraverbal (el tono, volumen,...de la voz) y no verbal. No es lo mismo hablar con la mirada puesta en los ojos de la otra persona para aseverar nuestra decisión en lo que decimos, que contestar a sus preguntas evitando su mirada, de forma huidiza. No será lo mismo tocar y aproximarnos al enfermo cuando hablamos, que hablar desde cierta distancia. Estos detalles que parecen que pasan desapercibidos a los pacientes, al enfermo en fase final le son muy clarificadores. La comunicación no verbal no debería estudiarse como una unidad aislada, sino como una parte inseparable del proceso global de comunicación. Puede servir para repetir, contradecir, sustituir, complementar, acentuar o regular la comunicación verbal ${ }^{5}$; pero tenemos que tener en cuenta, que cuando el lenguaje de nuestro cuerpo contradice de forma clara lo que decimos, tiene mayor peso para quien lo recibe el lenguaje no verbal. A todos nos ha pasado alguna vez, que hemos quedado con alguien para vernos dentro de unos días y aunque el interlocutor nos ha dicho que iba a ir, tenemos la seguridad de que no iba a presentarse a dicha cita y efectivamente no acudir. Su forma de decirlo, sus gestos, su expresión corporal nos estaba confirmando la contradicción entre lo que decía y como lo decía. La comunicación no verbal nos delata sin saberlo.

\section{CUIDAR CON CUIDADO}

Siempre hemos oído "ten cuidado", "baja con cuidado", "sube con cuidado",...desde nuestra infancia, en la adolescencia, en nuestra vida profesional ¿cuantas veces se nos indicado, ¡cuidado!?

Cuando en nuestra profesión hemos adoptado el cuidado como paradigma el concepto del mismo ha ido cambiando y adaptándose a cada una de las circunstancia de nuestra vida profesional. En esta reflexión sobre el cuidado al paciente en estado terminal, queremos hacer nuestra la frase: “... con cuidado” y transformarla en el objetivo de nuestro aporte: cuidar con cuidado y no solo como un 
juego de palabras, sino como el centro de nuestro quehacer con el paciente en estado terminal.

La importancia del Equipo interdisciplinar en la asistencia sanitaria es vital en todos los ámbitos, pero en el área de los Cuidados Paliativos, el Equipo es el eje que genera todo el apoyo al Paciente. Los enfermeros, como miembros de ese equipo, en esta fase final vamos a seguir siendo los brazos ejecutores de los cuidados cotidianos; quizás simples, pero no sencillos, esos que el enfermo y la familia no pueden realizar; el primero porque poco a poco vaya perdiendo sus capacidades físicas y su autonomía en el autocuidado y los segundos porque o bien el paciente se encuentre ingresado en una institución y sean los profesionales de esa institución los encargados de realizarlos ó están en una fase de incapacidad emocional ó claudicación, para poder hacerlo. En esas circunstancias las enfermeras* somos los profesionales responsables de los cuidados cotidianos: la higiene, la nutrición, la eliminación, la oxigenación,.... la cobertura mas primaria de las necesidades básicas del ser humano; que forman parte de la parcela del autocuidado que la persona realiza durante su etapa vital y por tanto es parte de su intimidad. El aseo y la eliminación son actividades propias y nadie suele estar presente en ambas, démonos cuenta de que podemos estar desnudos delante de otras personas, pero buscamos la más absoluta soledad para realizar estas otras. Pues bien, el paciente en estado final puede perder el control de sus esfínteres y los cuidadores vamos a ser partícipes de esa pérdida. Aquí nuestro cuidado debe ser doble, debemos realizar nuestra acción profesional con cuidado, es decir, con el empeño suficiente para no dañar y no molestar, pero también con la suficiente discreción de no invadir su intimidad más de lo necesario, de no violentar su parcela más íntima y propia. Debemos cuidar con cuidado. Como dice Simone de Beauvoir:

“..., tenía simpatía por las enfermeras, las sentía ligadas a ella, por la familiari- dad de las tareas humillantes para ella, quizás repugnantes para ellas; el interés que le demostraban tenía por lo menos apariencia de amistad, le daban confianza, le calmaban, sin aparentar nunca superioridad" 6 .

\section{CUIDANDO LA INTIMIDAD CON CUIDADO}

Existen condicionantes que determinan la relación necesaria para que surja la intimidad del cuidado, estos atributos son la confianza, es decir la virtud por la que el usuario confía en mí y para el que tengo deber de secreto de lo que me deposita y que requiere de mí, competencia y capacidad de comunicación y escucha. La comprensión, que va más allá de la empatía, se basa en la credibilidad de la enfermera ante el paciente.

La intimidad del cuidado se caracteriza porque se apoya en una relación de proximidad, con lo que el usuario se ve obligado a ponerse al descubierto para la aplicación de acciones de cuidado terapéutico, y confía en que la enfermera entre en su intimidad desde la confidencialidad. Aparece por tanto, la necesidad de guardar un espacio intimo, tanto en lo físico como en lo relacional, para ser utilizado únicamente para el cuidado.

Debemos tener siempre presente otro aspecto de la intimidad, lo íntimo, lo que no se muestra y constituye lo más propio, marcando la propia libertad que acompaña al ser humano durante toda la vida y en los momentos de su muerte.

Intimidad, por tanto, tendría ahora una acepción distinta, es como la parte reservada, lo que se guarda para uno, lo que le lleva a encontrar la felicidad, aquello que se refiere a lo más particular de los pensamientos, de los afectos ocultos, los interiores de una persona; el que cuida, no entra en lo íntimo de la persona visto desde este enfoque, pero debe saber de su existencia para respetarlo.

Así tendríamos dos aspectos de la intimidad:

\footnotetext{
* En este texto se emplea indistintamente la palabra enfermera, enfermero, cuidador,...
} 
- El cuidado de la persona con la debida atención, sensibilidad y respeto a su intimidad. En este sentido se refiere a la intimidad del cuidado que tiene que ver con descubrirse físicamente al otro en un espacio y momento concreto, es decir, poner todos los medios para cuidar en la intimidad y con intimidad.

- La protección de lo intimo de la persona cuidada, lo que incluye no revelar aspectos de la intimidad conocidos desde el cuidado y también, en un plano más activo, la enfermera ayuda a la persona desde la planificación del cuidado para mantener las barreras para preservar lo que les es intimo y no quiere que se conozca.

Entre ambos sentidos estaría quizás la idea de la discreción, en cuanto no hurgar en la intimidad más de lo que para el cuidado se precise o la persona nos quiera trasmitir.

\section{LA TÉCNICA COMO MEDIO DEL CUIDADO CON CUIDADO}

Con relación a las técnicas nuestro doble cuidado debe ser la indicación de la técnica a realizar: Lo que puede ser hecho, a veces, no tiene que ser hecho. Debemos evaluar y pensar antes de actuar.

La función fundamental del cuidado al paciente en estado terminal es proporcionar los cuidados de confort y de calidad de vida, a través de la valoración continua de las Necesidades Básicas propuestas por Virginia Henderson ó de los Patrones de Gordon o cuales quiera sea la metodología de valoración enfermera asumida por el Equipo Interdisciplinario, se deberá realizar un diagnostico enfermero de la situación del paciente, para poder definir un riguroso plan de cuidados que nos permita dar soluciones o paliar en parte las molestias y el sufrimiento del enfermo. Ese debe ser nuestro objetivo: el confort y el bienestar del paciente, dentro de lo posible, garantizando una continuidad y revisión permanente del plan de cuidados definido.

La valoración tendrá que basarse en los tres parámetros fundamentales: observación, la entrevista (si es posible) y sobre todo en la evaluación continua: debiendo monitorizar los síntomas, establecer un seguimiento de cada uno de ellos, y evaluar continuamente, si se resuelven, si no se resuelven, en resumen, evaluación constante de cada una de las actividades que llevamos a cabo. No perder nunca nuestro objetivo: cuando un paciente esta diagnosticado de una enfermedad en fase terminal, nuestro objetivo no es curar, es cuidar; nuestro objetivo es el confort y mantener la mayor calidad de vida; no es la supervivencia lo que importa, sino la calidad de esa supervivencia; en otras palabras: no es dar días a la vida, sino vida a los días que le queden antes de morir.

\section{NECESIDADES BÁSICAS Y/O PATRONES A EVALUAR}

Trabajar con patrones y/o necesidades básicas en Enfermería es una metodología muy difundida para establecer unos correctos cuidados. Existen patrones de valoración de necesidades, de diversas escalas, pero en el paciente aquejado de una enfermedad terminal, el concepto prioritario es la Calidad de Vida:

"La calidad de vida se define como la percepción del individuo sobre su posición en la vida dentro del contexto cultural y el sistema de valores en el que vive y con respecto a sus metas, expectativas, normas y preocupaciones. Es un concepto extenso y complejo que engloba la salud física, el estado psicológico, el nivel de independencia, las relaciones sociales, las creencias personales y la relación con las características sobresalientes del entorno"

Como vemos la calidad de vida es un concepto que aunque tiene en cuenta lo biológico, en lo que hace más hincapié es en lo biográfico, depende de cada individuo y por lo tanto tiene mucho de subjetivo.

\section{FUNCIONES ESPECÍFICAS DEL CUIDADO}

Función cuidadora. El desarrollo del cuidado al paciente con una enfermedad 
terminal, debemos contemplarlo en tres vertientes:

- cuidados básicos generales: encaminados a proporcionar al paciente el máximo confort en cada momento (cuidados de la piel, eliminación, reposo, sueño, ....)

- cuidados preventivos: su fin es evitar los problemas que suelen desarrollar con frecuencia estos pacientes (bucales, estreñimiento, sangrados y entre los emocionales: miedo, ansiedad, tristeza, ....)

- cuidados sintomáticos: son aquellos que se realizan para intentar resolver o paliar los estados patológicos que van apareciendo (micosis, úlceras, anorexia, constipación abdominal, disnea, ....)

\section{Función protectora o de garante}

- Mantener un ambiente seguro, se encarga de prevenir lesiones y protege al paciente de efectos adversos relacionados con el tratamiento, las técnicas, etc.

- Defiende los derechos humanos y legales del paciente y le ayuda a ejercer esos derechos en caso necesario. Teniendo el horizonte del Principio de Autonomía, como eje del cuidado.

La realidad del cuidar con cuidado al paciente que sufre una enfermedad terminal debe fundamentarse en el aprecio y reconocimiento de la dignidad y valor de la persona enferma.

¿Qué se puede aprender del cuidado a una persona en fase terminal de su enfermedad y cercana a la muerte?

Siguiendo el apartado de los cuidados enfermeros del libro Avances de los Cuidados Paliativos ${ }^{8}$ :

- A descubrirlos, superando nuestros prejuicios y temores ante el proceso de morir y la muerte.

- A escuchar, no solo a oír, a darles la información adecuada a la que solicitan, sin mentiras añadidas.

- A estrechar la mano hasta el final.

- A permanecer serenos frente al sufrimiento y la muerte.

- A reflexionar y pensar en los que nos resultaron difíciles de soportar y cuya muerte nos causó alivio.
De la misma manera que damos, recibimos y si estamos en disposición para ello, nos enseñarán lo que necesitamos aprender, para lograr "cuidar con cuidado".

Con relación al cuidado final al paciente agónico, tengamos muy presente que aunque disminuyan los sentidos, no debemos dejar de hablar a la persona y por supuesto debemos seguir tocándole, acariciándole y no dejándole solo. El tacto es muy importante, ya que proporciona la unión con la persona, el último eslabón del contacto humano. Aunque el paciente no pueda responder de forma verbal, puede responder de otras formas: cierre de una mano, de los ojos, emisión de lágrimas, ... Cosas tan obvias y tan sencillas como permanecer alrededor de la persona, sentados a la misma altura y mirándole a la cara, favorecen la comodidad de todos, pero sobre todo de la persona agónica. Hablar en tono suave, claro, evitando ruidos y sobre todo evitando conversaciones ajenas al paciente.

El cuidado con cuidado conlleva hacer sentir a la persona agónica que "estamos ahí”, acompañándole en el último tramo de si vida.

Un aspecto que a veces pasa desapercibido es el cuidado hacia los familiares, y/ó hacia el paciente de la cama de al lado. Quizás sólo sea necesaria nuestra presencia, un apretón de manos, una caricia; en esos momentos las palabras pueden resultar inapropiadas o inoportunas...

Como resumen de lo anteriormente expuesto queremos trasmitir que para el cuidado a la persona con una enfermedad en fase terminal, independientemente del ámbito de actuación, hay una vertiente básica con dos aspectos básicos que tienen que estar presentes:

- El enfoque integral de la persona enferma, en el que hay que abarcar los aspectos: físicos, emocionales, psicológicos, sociales y espirituales de la misma.

- El apoyo a la familia y/o entorno afectivo, como instrumento indispensable para la prestación de cuidados y a la que hay que cuidar de la misma manera para 
ayudarles y evitar la claudicación emocional y a veces también física.

\section{LA RESPONSABILIDAD MORAL EN EL CUIDADO AL PACIENTE EN SITUACIÓN TERMINAL}

Iniciamos este aspecto del "cuidar con cuidado" al Paciente en estado terminal, con una introducción global sobre la "responsabilidad moral": porque cuando hablamos de "responsabilidad moral" en el ámbito de la asistencia sanitaria, ¿nos referimos todos al mismo concepto? ¿qué es "responsabilidad moral"?.... La Ética de los cuidados, se ha basado históricamente, en el patrón especifico de la "ética de la sumisión", siendo su principio básico, la obediencia ciega a la autoridad profesional, tanto en el orden técnico como en el orden moral, del médico, de la institución, de las jerarquías religiosas, etc.

La ética tradicional del cuidado se sustentaba en una serie de virtudes, reales ó imaginarias, como la sumisión, la fe, la caridad, ... pero sobre todo, como antes se ha dicho la "obediencia a la autoridad", se esperaba que la cuidadora tuviera "voluntad pero no decisión", "acción pero no pensamiento". A lo largo de la historia no ha tenido una ética autónoma, sino heterónoma: la autoridad y la responsabilidad, la tenía el "dirigente" (médico, órdenes religiosas, etc.) y, por tanto, la responsabilidad de la enfermera no existía como propia.

Desde hace más de siglo y medio, la enfermería ha pasado por diferentes etapas, algunas caminando hacia delante y otras mas o menos hacia atrás, pero lo cierto es que en el alba del siglo XXI, la profesión está consolidada y emergiendo hacia una autonomía real, formando parte de equipos asistenciales, con una equidistancia horizontal y por tanto, ya no puede basar su ética en la sumisión, ni en la ética de la convicción, sino que debe asumir la ética de la responsabilidad y ello conlleva asumir su responsabilidad moral ante el ser cuidado.

Parece necesario, por tanto, ahondar en el concepto de responsabilidad. Según Ingarde 1980, en su sentido más básico se refiere: "alguien es consciente de una conducta y/ó de una acción, que asume como propia, así como de las consecuencias que de ella se derivan".

Para que se pueda asumir la responsabilidad es preciso que confluyan, al menos, tres criterios según H. Tam, 1981:

- Libertad: el sujeto debe actuar de manera libre.

- Conducta intencional: debe ser consciente de lo que esta realizando.

- Acción u Omisión: el sujeto realiza una acción ó deja de realizar algo.

La Responsabilidad Moral, constituye la forma primaria de responsabilidad y se caracteriza por tener como juez ó instancia última, la propia conciencia moral, guiada por los principios de uno mismo, viéndonos abocados a rendir cuentas de nuestros actos ante nosotros mismos, ante nuestra conciencia y nuestros valores.

Ante el Paciente en situación terminal, y/o agónico, la responsabilidad moral del cuidador, siempre compartida e inmersa en el equipo asistencial, es un claro ejemplo de "la propia conciencia moral", donde las decisiones y actividades, nacen, no sólo de su profesionalidad, sino de lo más íntimo de sus convicciones. Es en este campo donde la responsabilidad moral encuentra los mayores problemas, cuando los intereses entran en conflicto y el cuidador debe asumir su compromiso con el paciente, antes incluso que la asunción las normas de la institución o a los requerimientos de otros profesionales. Es en esas situaciones cuando para lograr el objetivo de cumplir con la responsabilidad moral hacia su paciente, debe adentrarse en el campo de la Ética de la Responsabilidad, para saber y poder ponderar todos los factores: convicciones y consecuencias.

Para finalizar con la reflexión sobre la responsabilidad moral, hacemos nuestra la visión de futuro sobre los cuidados paliativos del profesor D. Gracia ${ }^{7}$ de que el cuidado a los pacientes en situación terminal exige la defensa de la idea de la "calidad de vida" frente a la idea de la "santidad de vida" basada en el enfoque de la "ética de la convicción", siendo ésta 
sustituida en el movimiento de los cuidados paliativos por la Ética de la Responsabilidad. Uno de los grandes retos en este ámbito del cuidado, es el saber enlazar el "cuidado-proximidad-confianza" con la "justicia-equidad-derechos-obligaciones".

\section{REFLEXIÓN FINAL}

Para terminar querríamos traer una poesía de León Felipe

\section{BERTUCA}

En tu agonía, amor.

¡CUÁNTO le costó a la muerte apagarte los ojos!

Sopló una vez, dos veces,

tres veces -ibien lo vi!-

y tus ojos siguieron encendidos.

Alguien dijo:

Ya no tiene ni sol ni sal en las venas

y los ojos no se le apagan.

Yo llegué a pensar que no se apagarían nunca,

que quedarían encendidos para siempre

como las alas de una mariposa de oro eternamente abiertas

sobre los despojos de la muerte.

$\mathrm{Al}$ fin todo se hundió...

y tu mirada se torció y se deshizo

en un cielo turbio y revuelto...

Y ya no vi más que mis lágrimas ${ }^{8}$.

Este poema apareció en su libro "El ciervo y otros poemas" en mayo de 1957. En él narra la pena y el dolor sufrido por el poeta en la agonía de su esposa. Eso sólo bastaría para traerlo aquí, pero la dedica- toria de ese libro es quizás más conmovedora y esclarecedora. Dice así:

A la señora LUCERo CARRAL DE PANI

No tengo otra cosa mejor para poner en tus manos que este libro herético y desesperado. Soy un viejo pobre y un pobre viejo. Perdóname esta doble pobreza, "Lucerito"..., y deja que te bese las manos, esas manos misericordiosas de santa que amortajaron a Berta y le cerraron los ojos.

Cerrar los ojos a una persona que ha fallecido y ayudar a vestirla para su último rito de paso, cuando los familiares estar partidos y superados por el dolor, también es cuidar con cuidado.

\section{BIBLIOGRAFÍA}

1. Tribuna Sanitaria $\mathrm{n}^{\mathrm{O}} 135$, marzo 2001.

2. Los cuidados del confort en el final de la vida: Responsabilidad enfermera. Coordinador: Juan Carlos Delgado Antolín, componentes Francisca Hernández Martín, Aurora Hernández Rivas y Amparo Nogales Espert. Asociación de Bioética Fundamental y Clínica, 21 de octubre de 2005.

3. MARTín BuBER (8-2-1878 a 13-6-1965). Filósofo, teólogo y escritor austríaco de origen judío y conocido por su filosofía del diálogo.

4. Marcos Gómez Sancho. Instituto Canario de Estudios y promoción Social y Sanitaria, 1994. Pág. 267.

5. MARK L. KNAPP. La comunicación no verbal, el cuerpo y el entorno. Ediciones Paidos, $5^{\mathrm{a}}$ Edición, pág. 42.

6. Marcos Gómez Sancho. Avances en Cuidados Paliativos Tomo III.

7. Gracia, Diego. "Como arqueros al blanco", Editorial Triacastela-Madrid, 2004.

8. LEÓN Felipe. El ciervo y otros poemas. Finisterre editores, México 1974. Pág. 61. 